\title{
Pensée utilitaire
}

Dans la dernière édition du BMS, l'éditorial [1] et l'article des Médecins en faveur de l'environnement [2] font part du risque de fermeture de la section «Santé et environnement» de l'OFSP. Il ne s'agit pas ici de débattre de la question de savoir si une telle mesure est juste ou non et, le fait étant, pourquoi. La lecture des deux articles me rappelle cependant un passage du roman d'Aldous Huxley «Time must have a stop». En 1944 déjà, il faisait philosopher son personnage principal, Sebastian, sur la curieuse logique que suivent les actes de l'espèce humaine. Pour des idéaux politiques ou «l'utopie d'un fumeur d'opium», des millions de vies humaines seraient sacrifiées, même si l'on peut fortement douter que le but visé par là puisse jamais se réaliser. Dans le domaine de la nature, par contre, l'avenir de tout le genre humain est mis en jeu pour un gain à court terme. En fait, dans des secteurs où l'on ne peut guère faire de prédictions, nous mettons le présent en grand danger pour le bien d'un avenir plus qu'incertain. Par rapport néanmoins à la nature où la prédiction est facile quant aux conséquences de notre comportement, nous faisons le sacrifice de l'avenir au profit d'une pensée à court terme.

Il se peut qu'on ait appris quelque chose depuis 1963, date de la mort de Huxley, sur la base de quelques amères expériences de catastrophes naturelles. Et néanmoins, la pensée d'Huxley m'apparaît toujours actuelle. A notre époque aussi, la valeur des revendications écologiques fluctue selon les intérêts et les données économiques et politiques. Des protocoles climatiques sont signés ou non, selon le cas, et l'on se contente de prendre acte du dépassement des valeurs limites des polluants, en se réservant de les adapter en cas d'urgence. «Notre pensée tend à se faire utilitaire», relève Hans Ruh, spécialiste en éthique sociale, dans son ouvrage paru en 1995 et intitulé «Elément perturbateur, l'être humain. Portes de sortie de la crise écologique» (trad. FMH). Il relève chez l'homme la «faculté éternelle» de vivre avec ses contradictions. Attitude logique face aux soucis et aux nécessités, mais qui peut se révéler catastrophique à long terme pour l'environnement. Selon Ruh, ce ne sont pas les suites écologiques au sens étroit du terme, mais les conséquences socioculturelles, éventuellement aussi politiques et économiques d'un comportement destructeur de l'environnement qui sont intolérables.

Et les conséquences sur la santé, pourrait-on ajouter. L'article cité au début affirme que selon l'Organisation mondiale de la santé, un tiers des maladies infantiles en Europe sont dues à des facteurs environnementaux. Dans ce cas aussi, l'estimation de Ruh est pertinente: «Sans conteste, les personnes les plus touchées par les catastrophes écologiques n'ont que peu participé à leurs causes». Sur cette toile de fond, il n'est que pure logique que le corps médical exige que la santé des enfants, en tant que groupe social particulièrement sensible, soit portée au premier plan des préoccupations face à notre environnement pollué. Le Conseil fédéral semble avoir d'autres priorités puisqu'il veut aussi renoncer au programme Environnement et santé des enfants.

\section{Bruno Kesseli}

1 Steiner-König U. Programme systématique d'abandon des tâches. Bull Méd Suisses 2006; 87(11):429.

2 Moll R, Biedermann A. Section santé en environnement de l'OFSP et plan d'action pour la santé des enfants: victimes des mesures d'économie de la Confédération. Bull Méd Suisses 2006; 87(11):450-1. 